\title{
Search for small-mass black-hole dark matter with space-based gravitational wave detectors
}

\author{
Naoki Seto* and Asantha Cooray ${ }^{\dagger}$ \\ Theoretical Astrophysics, Division of Physics, Mathematics and Astronomy, California Institute of Technology, MC 130-33, \\ Pasadena, California 91125, USA
}

(Received 11 May 2004; published 15 September 2004)

\begin{abstract}
If the halo dark matter were composed of primordial black holes (PBHs) with mass between $10^{16}$ and $10^{20} \mathrm{~g}$, their gravitational interaction with test masses of laser interferometer may lead to a detectable pulselike signal during the fly-by. If a proof-mass noise of $3 \times 10^{-15} \mathrm{~m} / \mathrm{s}^{2} / \mathrm{Hz}^{1 / 2}$ down to $\sim 10^{-5} \mathrm{~Hz}$ is achieved by the Laser Interferometer Space Antenna, the event rate, with signal-to-noise ratios greater than 5 , could become $\sim 0.1$ per decade, involving black holes of mass $\sim 10^{17} \mathrm{~g}$. The detection rate could improve significantly for future space-based interferometers, though these events must be distinguished from those involving perturbations due to near-Earth asteroids. While the presence of primordial black holes below a mass of $\sim 10^{16} \mathrm{~g}$ is now constrained based on the radiation released during their evaporation, the gravitational-wave detectors could potentially extend the search for PBHs to several orders of magnitude higher masses.
\end{abstract}

DOI: 10.1103/PhysRevD.70.063512

PACS numbers: 95.35.+d, 95.55.Ym, 97.60.Lf

Based on observed rotational velocity measurements of the Milky Way disk, the presence of a substantial dark matter component in the Milky Way halo is now well established [1]. With no unique guidance as to the nature of dark matter, a large number of candidates based on both astronomical arguments, such as baryonic dark matter involving substellar mass remnants [2], and particle physics expectations [3] are now routinely considered to explain the missing mass. An interesting possibility is that the halo dark matter is composed of primordial black holes (PBHs) [4]. While PBHs are expected over a rather wide range of masses [5], the population below $\sim 5 \times$ $10^{14} \mathrm{~g}$ is expected to have evaporated by the Hawking radiation over the age of the Universe [6], while holes with a mass slightly above this limit are still emitting high energy particles today [7]. With observations related to the galactic $\gamma$-ray background, the evaporating blackhole contribution to the dark matter density in the Universe, in terms of the critical density, is at the level roughly below $10^{-8}$ [7].

From the high mass end, constraints on the possibility that dark matter is composed of PBHs come from dynamical arguments such as the potential disruption of galactic clusters and similar bound structures [8]. These constraints generally limit the primordial black-hole mass to be roughly below a few solar mass. Similar constraints come from galactic microlensing observations, which limit primordial black-hole masses to be below roughly $10^{-7} M_{\odot}$ [9]. In combination, at least over a $10^{9}$ decade in a primordial black-hole mass, between evaporating holes with mass below $10^{17} \mathrm{~g}$ and $\sim 10^{-7} M_{\odot}$, remains yet to be studied and techniques to understand their presence are limited (see also [10]). Though the galactic $\gamma$-ray background and its weak anisotropy sug-

\footnotetext{
*Email address: seto@caltech.edu

†Email address: asante@ caltech.edu
}

gest an evaporating PBH density of roughly $10^{10} \mathrm{pc}^{-3}$, PBHs can explain the total halo dark matter if the $\mathrm{PBH}$ mass spectrum enhances the abundance above $10^{17} \mathrm{~g}$ [11]. A promising possibility of probing small-mass PBHs involves femtolensing of $\gamma$-ray bursts (GRBs) both in terms of an interference pattern in the frequency spectrum [12] or relative flux differences in the astronomical unit scale separated light curves [13]. The existing data weakly constrain the mass density fraction, relative to the critical density, of compact objects with mass between $10^{17}$ and $10^{20} \mathrm{~g}$ to be below 0.2 , if the average redshift of GRBs is unity [14]. This technique constrains only the extragalactic density, though, it would be interesting to see if the prediction [11] related to the galactic dark matter can directly be tested.

The PBHs, especially at the small-mass end, are not expected to interact or be captured by other massive bodies such as the Sun; they can be considered as another weakly interacting massive particle (WIMPs). In contrast to active PBHs with masses $\lesssim 10^{15} \mathrm{~g}$, the physical nature of PBHs with $\gtrsim 10^{17} \mathrm{~g}$ is characterized only by their masses and extremely small size. In reality, they can be detected only through the gravitational interaction. But, as their masses are much larger than standard WIMPs predicted by particle physics, the expected flux of PBHs would be very small and we need detectors with a large effective area for their search.

In this paper, we suggest that PBHs around a mass of $10^{17} \mathrm{~g}$ could in fact be detected directly with space-based gravitational-wave detectors. These detectors have large cross sections to moving massive bodies that would not be realized with other tools. These missions include the Laser Interferometer Space Antenna (LISA) [15] and some other missions of the future that are now starting to be discussed in the literature. The direct detection simply involves the gravitational interaction between the fly-by PBHs and detector test masses such that with 
high sensitivity even a small gravitational perturbation can eventually be seen above the detector noise. As an example, the LISA mission is expected to probe the frequency range between $10^{-5}$ and $10^{-1} \mathrm{~Hz}$ and detect perturbations above $h \sim \delta L / L \sim 10^{-21}$ at $\sim 1 \mathrm{mHz}$ with detectors separated by arm lengths $L \sim 5 \times 10^{6} \mathrm{~km}$.

The interaction of a PBH and a gravitational-wave observatory test mass can be considered either as a direct interaction, when the fly-by separation is smaller than the arm length, or as a tidal interaction, in the case where the arm length is smaller than the fly-by distance. We first consider the former situation and take the scenario with a PBH mass $M$. passing by one of the test masses of the interferometer with a relative velocity $V$ with the closest approach distance $R$. The time dependent acceleration $a(t)$ of the spacecraft test mass towards the direction of the closest approach has a single pulselike structure given by

$$
a(t)=\frac{G M_{\bullet} R}{\left[R^{2}+(V t)^{2}\right]^{3 / 2}},
$$

where we have set the origin of the time coordinate, $t=$ 0 , to be at the closest approach. Note that the fly-by PBH can also perturb other test masses of the interferometer configuration since at least three or more spacecrafts are generally utilized in future gravitational-wave observatories. When making an order-of-magnitude estimate we neglect additional information related to such perturbations and ignore aspects that involve the relative configuration of interferometer-body system related to the perturbing $\mathrm{PBH}$.

The Fourier component $a(f)$ of Eq. (1) is given in terms of the 1st order modified Bessel function $K_{1}$ as

$$
a(f)=\int_{-\infty}^{\infty} d t e^{2 \pi i f t} a(t)=2 \frac{G M \bullet}{R V} K_{1}[2 \pi f R / V] .
$$

For the direct detection, the signal-to-noise ratio (SNR) of the pulse $a(t)$ is formally written as $\mathrm{SNR}^{2}=$ $2 \int_{0}^{\infty} a(f)^{2} / a_{n}(f)^{2} d f$ with the noise spectrum $a_{n}(f)$ of the detector. In the case of space-based interferometers, the noise has two main contributions involving the proofmass noise and the optical path noise with the former dominating the low-frequency regime. As we will soon discuss, the typical signal $a(f)$ due to the fly-by PBH of mass around $M_{\bullet} \geq 10^{17} \mathrm{~g}$ has support in the SNR integral when $f \leqq 10^{-4} \mathrm{~Hz}$. This is in the low-frequency regime of all the proposed future interferometers, including the LISA mission. The proof-mass noise of LISA is estimated to be constant down to $\sim 10^{-4} \mathrm{~Hz}$. It might become a factor of 2 larger at $3 \times 10^{-5} \mathrm{~Hz}$ and more at lower frequencies [16], however, the low-frequency behavior of the noise curve, around $10^{-5} \mathrm{~Hz}$, is yet to be determined precisely. In order to estimate the SNR, we simply extrapolate the constant proof-mass noise down to $f=0$, and set $a_{n}(f)=2 a=$ const, where $a$ is the proofmass noise for a single inertial sensor in various interfer- ometers. While frequency dependence of the proof-mass noise is ignored, this dose not mimic the reality of the detectors. We do pay attention to the frequency dependence of the fly-by pulse event so as to understand requirements on the low-frequency behavior of the proofmass noise.

After some straightforward algebra, we obtain the SNR as

$$
\mathrm{SNR}^{2}=\frac{3 \pi}{32} \frac{\left(G M_{\bullet}\right)^{2}}{V R^{3} a^{2}} \quad(R<L) .
$$

Note that this relation corresponds to the second formula of Eq. (4.35) in Ref. [15], but our result differs by $1 / 13$ $8 \%$ due to the exact use of a limit associated with the Bessel function, instead of an approximation used there, and after taking in to account a difference in the definition related to the proof-mass noise.

Since the above expression (3) is for the case where the closest approach distance $R$ to a single spacecraft is smaller than the arm length $L$ of the interferometer, namely, $R<L$, we now consider the opposite case with $R>L$. Now, the tidal deformation of the interferometer is a measurable effect, but this involves an additional suppression factor $\sim n(L / R)$ in Eq. (1), where we have ignored the numerical prefactor $n$ as we do not consider detailed geometry of these events, though we note that this factor could be greater than unity (up to 2 ) in certain favorable configurations of the interferometer with respect to the trajectory of the PBH fly-by. In this tidal limit, we find the SNR to be

$$
\mathrm{SNR}^{2}=\frac{3 \pi}{32} \frac{(G M . L)^{2}}{V R^{5} a^{2}} \quad(R>L) .
$$

This is the relevant expression for LISA in the interesting mass range $M_{\bullet} \gtrsim 10^{17} \mathrm{~g}$, though, at the low mass end $\left(10^{16} \mathrm{~g}\right)$, the former expression applies for LISA.

Using Eq. (4) we can now estimate the maximum length of the closest approach $R_{\max }$ for a given SNR threshold

$$
\begin{aligned}
R_{\max }= & 5.3 \times 10^{11}\left(\frac{M \cdot}{10^{17} \mathrm{~g}}\right)^{2 / 5}\left(\frac{V}{350 \mathrm{~km} / \mathrm{s}}\right)^{-1 / 5}\left(\frac{\mathrm{SNR}}{5}\right)^{-2 / 5} \\
& \times\left(\frac{a}{a_{0}}\right)^{-2 / 5}\left(\frac{L}{L_{0}}\right)^{2 / 5} \mathrm{~cm}
\end{aligned}
$$

where $a_{0}=3 \times 10^{-15} \mathrm{~m} / \mathrm{s}^{2} /(\mathrm{Hz})^{1 / 2}$ and $L_{0}=$ $5 \times 10^{11} \mathrm{~cm}$ are the reference fiducial parameters for LISA in its current design [15] (at least the proof-mass noise around $f \sim 10^{-4} \mathrm{~Hz}$ ). We have taken the typical velocity dispersion $V=220 \sqrt{5 / 2}=350 \mathrm{~km} / \mathrm{s}$ of the halo dark matter particles relative to the solar system using the galactic rotation velocity of $220 \mathrm{~km} / \mathrm{s}$ and the galactic radius to the solar system of $r_{g} \sim 8 \mathrm{kpc}$ following Ref. [8]. The gravitational perturbation involves a pulselike signal in the data streams with a characteristic fre- 
quency given by

$$
\begin{aligned}
V / R_{\max } \sim 6.4 \times 10^{-5}\left(\frac{M \cdot}{10^{17} \mathrm{~g}}\right)^{-2 / 5}\left(\frac{V}{350 \mathrm{~km} / \mathrm{s}}\right)^{6 / 5} \\
\times\left(\frac{\mathrm{SNR}}{5}\right)^{2 / 5}\left(\frac{a}{a_{0}}\right)^{2 / 5}\left(\frac{L}{L_{0}}\right)^{-2 / 5} \mathrm{~Hz} .
\end{aligned}
$$

As mentioned already, for most planned space interferometers such as LISA, such a low frequency is in the regime where the detection is limited by the proof-mass noise.

When we assume that the halo dark matter with density $\rho_{D M} \sim 0.011 M_{\odot} / \mathrm{pc}^{3}$ [17] around the Sun is made with PBHs of mass $M_{\bullet}$, their flux, $F=\rho_{D M} V M_{\bullet}^{-1}$, is

$$
\begin{aligned}
F= & 9.0 \times 10^{-27}\left(\frac{\rho_{D M}}{0.011 \mathrm{M}_{\odot} \mathrm{pc}^{-3}}\right)\left(\frac{V}{350 \mathrm{~km} / \mathrm{s}}\right) \\
& \times\left(\frac{M \bullet}{10^{17} \mathrm{~g}}\right)^{-1} \mathrm{~cm}^{-2} \mathrm{yr}^{-1},
\end{aligned}
$$

and the fly-by event rate, $\dot{\eta}=\pi F R_{\max }^{2}$, above a certain SNR is

$$
\begin{aligned}
\dot{\eta}= & 0.01\left(\frac{M_{\bullet}}{10^{17} \mathrm{~g}}\right)^{-1 / 5}\left(\frac{\mathrm{SNR}}{5}\right)^{-4 / 5}\left(\frac{V}{350 \mathrm{~km} / \mathrm{s}}\right)^{3 / 5} \\
& \times\left(\frac{\rho_{D M}}{0.011 M_{\odot} \mathrm{pc}^{-3}}\right)\left(\frac{a}{a_{0}}\right)^{-4 / 5}\left(\frac{L}{L_{0}}\right)^{4 / 5}\left(\frac{N}{1}\right) \mathrm{yr}^{-1},
\end{aligned}
$$

where $N$ represents the effective number of interferometers with sufficient relative distances. While LISA involves a single set of interferometers, some proposed space missions plan to use multiple interferometer arrays with large separation to improve the localization of binary sources such that $N \geq 2$. If the signal is to be extracted using a correlation analysis, especially for low signal-to-noise events, of two arrays, there is no further increase in the event rate and one should continue to use $N=1$.

For the reference parameters of LISA, assuming a useful SNR threshold of 5 , the detection rate is $\sim 0.1$ per $10 \mathrm{yr}$ as shown in Eq. (8). In the tidal limit, the event rate weakly depends on mass $M_{\bullet}$ by $\dot{\eta} \propto M_{\bullet}^{-1 / 5}$. The combination $\left(a L^{-1}\right)$ that appears in all of the above expressions determines the low-frequency sensitivity of the interferometers to the gravitational-wave amplitude $h$. Therefore, once the threshold distances $R_{\max }$ are confirmed to be larger than the arm length $L$, we can easily compare the event rate for various interferometers by studying their noise curves at the low-frequency regime. In the case of LISA, when the mass $M$. falls below $10^{17} \mathrm{~g}$, the maximum fly-by distance approaches that of the arm length $\left(\sim 5 \times 10^{11} \mathrm{~cm}\right)$. In this case, we repeat the event rate using the SNR involving the direct perturbation [Eq. (3)]; in this limit, for reference, the event rate depends on the mass $M_{\bullet}$ by $\dot{\eta} \propto M_{\bullet}^{1 / 3}$.

In Fig. 1, we show the potential detectability of fly-by events involving $\mathrm{PBHs}$ with mass $M_{\text {• using various }}$

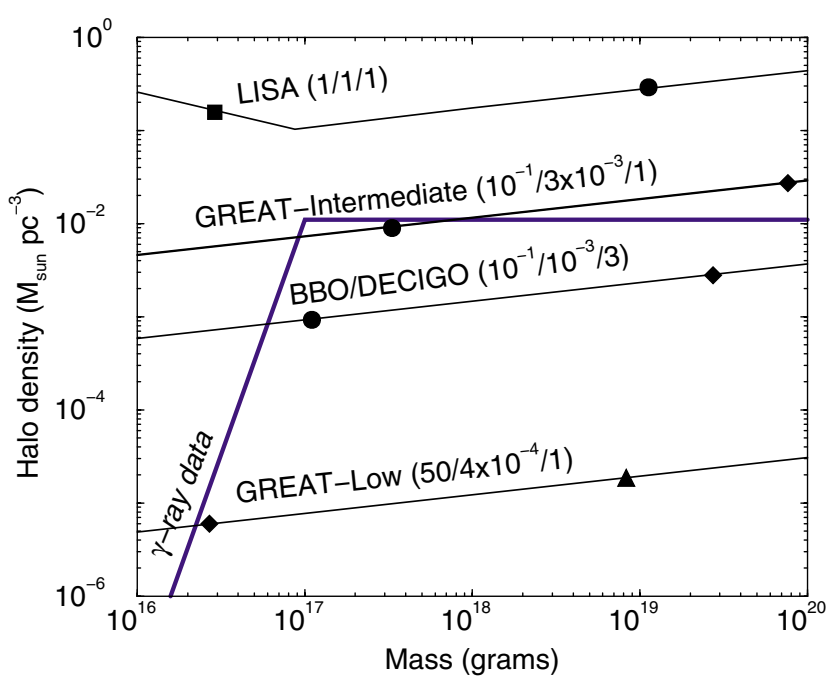

FIG. 1 (color online). The detection thresholds for PBH fly-by with various upcoming space-based gravitational-wave observatories (in solid lines from top to bottom: LISA, GREAT intermediate-frequency mission [18], BBO/DECIGO [19], and GREAT very-low-frequency mission). The thick solid line shows the expected density of halo dark matter in the form of black holes; at $M_{\bullet} \simeq 10^{15} \mathrm{~g}$, the density is constrained by $\gamma$-ray background observations such that the particle density is below $\sim 10^{10} \mathrm{pc}^{-3}$, while no similar constraints exist above $M_{\text {. }} \gtrsim 10^{17} \mathrm{~g}$ and we take the whole halo to be formed of black holes with the mass given in the horizontal axis (with a density $\left.0.011 M_{\odot} \mathrm{pc}^{-3}\right)$. The detection limits assume an event rate of a single detection per decade; if no events are detected, these curves would roughly correspond to the constraint one can put on the black-hole contribution to the halo dark matter density. In each of the instruments considered, we label the following three parameters: (arm length/proof-mass noise/total number of detector arrays) relative to those reference values of LISA (arm length: $5 \times 10^{6} \mathrm{~km}$, proof-mass noise: $3 \times$ $\left.10^{-15} \mathrm{~m} / \mathrm{s}^{2} / \sqrt{\mathrm{Hz}}\right)$. We assumed constant proof-mass noise down to $f=0$. The combination for BBO/DECIGO is chosen to reduce the strain sensitivity at low-frequency regime (but, not an optimal choice for measurement of the stochastic gravitational-wave background). The parameters come from the "BBO Grand" design of the recent concept study proposal [25]. The symbols on each of the curves represent the average frequency of the gravitational perturbation produced on the interferometer by a black hole of corresponding mass with the triangle, diamond, circle, and square representing frequencies of $10^{-7}, 10^{-6}, 10^{-5}$, and $10^{-4} \mathrm{~Hz}$, respectively. In the case of LISA, the threshold distance $R$ equals to the arm length $L$ at $M_{\text {. }}=0.87 \times 10^{17} \mathrm{~g}$ where the slope of the curve changes. The results for $\mathrm{BBO} / \mathrm{DECIGO}$ and GREAT-low missions are given for a reference. Their target frequency $\sim 0.1 \mathrm{~Hz}$ is largely different from the $\mathrm{PBH}$ search.

planned space-based interferometers. Instead of the event rate, our results are presented in terms of the local halo density of PBHs required for a detection, with SNR greater than 5, of one event per decade. For reference, to put these detections in the context of the galactic dark 
matter density, we also show current observational constraints for the PBH density from the $\gamma$-ray background $\left(M_{\bullet} \lesssim 10^{17} \mathrm{~g}\right)$ [11] and the expected density of dark matter based on a galactic model $\left(M_{\bullet} \gtrsim 10^{17} \mathrm{~g}\right)$ where the density is now determined to be $(0.011 \pm$ $0.005) M_{\odot} \mathrm{pc}^{-3}$ [17]. In the case of LISA, the transition $(R=L)$ between Eqs. (3) and (4) occurs at $M_{\bullet}=0.87 \times$ $10^{17} \mathrm{~g}$, but for all other interferometers, we find $R>L$ in the mass range considered in Fig. 1.

In Fig. 1, next to the name of each of these interferometers, we also denote three basic numbers that characterize an interferometer (arm length/proof-mass noise/number of detector arrays) normalized by the reference values for LISA.

As we mentioned, the low-frequency end of the LISA noise has not been finalized and there are strong scientific reasons to improve it beyond what was originally suggested [16]. The above rate for LISA 0.1 per decade is given for the constant proof-mass noise $3.0 \times$ $10^{-15} \mathrm{~m} \mathrm{~s}^{-2} \mathrm{~Hz}^{-1 / 2}$. If it becomes twice larger around $3 \times$ $10^{-5} \mathrm{~Hz}$, the event rate would decrease by a factor of $\sim 1.7$ for PBHs with $M_{\bullet} \sim 10^{17} \mathrm{~g}$. We also note here that if the event rate is too high with $\gtrsim 100 \mathrm{yr}^{-1}$, as would be the case for GREAT-low-frequency mission [18], the signals will be overlapped in frequency space due to the low-frequency nature. This will complicate the extraction and would involve a detailed analysis similar to the ones proposed to extract individual gravitational waves in their confusion limit. In this figure, we also note the characteristic frequency of the fly-by event. This is done to emphasize the very-low-frequency aspect of the detection, especially with respect to the noise requirements associated with proof mass, or acceleration noise.

Recently, some plans have been discussed around the intermediate band $(0.1$ to $1 \mathrm{~Hz})$ for a detection of stochastic wave background from inflation $[19,20]$. As a reference, we also plot the sensitivity curve of these missions under the simple assumption of a constant proof-mass noise down to very-low frequency. However, the relevant frequency for a $\mathrm{PBH}$ event is lower than the main target bands of these missions by more than 3 orders of magnitude. Thus, an enormous experimental effort would be required to keep good proof-mass noise over such a large dynamic range. Therefore, we believe that an extension of these intermediate missions for a PBH search would be quite a hard task from an experimental point of view, unless a significant progress in technology allows easy control of noise by the time these missions are flown.

In the search for fly-by PBHs, it is important to distinguish their signals from other signals in the interferometer. The primary targets of a space-based interferometer are low-frequency gravitational waves. In general, their measurement is limited to some extent by the astrophysical confusion noise, depending on the frequency. For example, the LISA detector noise is expected to be dominated by the galactic white dwarf binary confusion noise in the frequency band between $0.1 \lesssim f \lesssim$ $3 \mathrm{mHz}$ [15]. At a lower frequency than this range, gravitational waves from merging massive black-hole binaries might form a confusion noise [21,22]. This noise could be a strong obstacle for the PBH search, though the magnitude of the total background or that of the residual after subtraction of identified sources has uncertainty at present. At least, LISA would provide important information for its estimation.

When we measure the local acceleration related to PBH fly-by events, however, we can largely reduce the relative contribution of the low-frequency gravitational waves (with wavelength $\lambda_{G W}>L$ ) in the data streams by taking a certain combination of the data stream now known as the Sagnac [23]. This data combination nearly cancels gravitational waves using the fact that they propagate with the speed of light, though it would still be affected by fly-by perturbations. Therefore, the separation of $\mathrm{PBH}$ signals with other gravitational-wave signals is not as severe as the confusion noise problem for gravitationalwave observations alone. For the realization of the Sagnac mode the three arms of the interferometer should be operated properly. It is true that this is another technical requirement for the control of the interferometers, but the PBH search would give another reason to pursue the Sagnac mode.

There is one source of confusion, however. This involves similar pulses produced during the passage of near-Earth asteroids close to detectors [15]. Given the expected flux of minor bodies in the solar system, as determined by various observational data, the asteroid perturbations are dominated by those at the high mass end between $10^{13}$ to $10^{15} \mathrm{~g}$, or sizes around a $\mathrm{km}$ or slightly less, with an event rate of the order $0.05 \mathrm{yr}^{-1}$ (the rates for smaller mass events are substantially less and can be ignored). Unfortunately, though the relative velocities of asteroid events are smaller $\left(\sim 30 \mathrm{~km} \mathrm{~s}^{-1}\right)$ than those involving PBHs, these events could have similar frequencies (as PBHs) due to differences in the maximum distance to which they can be detected. On the positive side, orbital parameters of roughly $10 \%$ of such near-Earth asteroids are already known while this fraction is soon expected to grow substantially with dedicated near-Earth asteroid search programs [24] such that during the operation of these gravitational-wave observatories, expected perturbations from asteroids can be $a$ priori determined. Moreover, we expect optical follow-up searches of all candidate events to further refine the sample and to confirm the compactness of perturbers. Detection of a fly-by pulse with no optical counterpart would be a minimum criteria for the selection of a PBH event. For this purpose, we need a systematic optical survey around the interferometric area above some magnitude threshold. With increasing sensitivity, such as the 
case with the GREAT-low-frequency mission, the event rate of asteroids increases such that they might become the primary confusion noise. Removal of this confusion noise requires independent estimates of the asteroid mass at the few percent level in addition to the orbit.

While with gravitational-wave detectors the presence of PBHs can be established, it is not easy to determine masses of individual events from a single pulse signal that is characterized mainly by two numbers: the amplitude, $M / R^{3}$, and the time scale, $R / V$, made from three variables $M, R$, and $V$. This is due to the fact that there is an unknown associated with the fly-by distance or the PBH trajectory. If multiple detectors are perturbed, one can establish the trajectory and then use that information to determine mass.

We thank members of the Caltech TAPIR group for helpful comments. This work has been supported by the Sherman Fairchild Foundation, DOE DE-FG 03-92ER40701 at Caltech (A.C.) and NASA Grant No. NAG5-10707 (N. S.).

Note added.-While this paper was under editorial review, we became aware of a similar paper on the same topic [26]. Their results are consistent with our calculations.
[1] S. M. Faber and J.S. Gallagher, Annu. Rev. Astron. Astrophys. 17, 135 (1979).

[2] V. Trimble, Annu. Rev. Astron. Astrophys. 25, 425 (1987).

[3] G. Jungman, M. Kamionkowski, and K. Griest, Phys. Rep. 267, 195 (1996).

[4] Ya. B. Zeldovich and I. D. Novikov, Sov. Astron. 10, 602 (1966); S. W. Hawking, Mon. Not. R. Astron. Soc. 152, 75 (1971); B. J. Carr and S.W. Hawking, Mon. Not. R. Astron. Soc. 168, 399 (1974).

[5] B. J. Carr, Astrophys. J. 201, 1 (1975); I. D. Novikov et al., Astron. Astrophys. 80, 104 (1979).

[6] S.W. Hawking, Nature (London) 248, 30 (1974); Commun. Math. Phys. 43, 199 (1975).

[7] D. N. Page and S.W. Hawking, Astrophys. J. 206, 1 (1976); J. H. MacGibbon and B. J. Carr, Astrophys. J. 371, 447 (1991).

[8] B. J. Carr and M. Sakellariadou, Astrophys. J. 516, 195 (1999).

[9] C. Alcock et al., Astrophys. J. Lett. 499, 9 (1998).

[10] T. Nakamura et al., Astrophys. J. 487, L139 (1997); K. T. Inoue and T. Tanaka, Phys. Rev. Lett. 91, 021101 (2003).

[11] D. B. Cline, Astrophys. J. Lett. 501, 1 (1998); E. L. Wright, Astrophys. J. 459, 487 (1996).

[12] A. Gould, Astrophys. J. Lett. 386, 5 (1992).
[13] R. J. Nemiroff and A. Gould, Astrophys. J. Lett. 452, 111 (1995).

[14] G. F. Marani et al., Astrophys. J. Lett. 512, 13 (1999).

[15] K.V. Danzmann et al., LISA Pre-Phase A Report No. MPQ-233, 1998, 2nd ed., http://lisa.jpl.nasa.gov

[16] P. L. Bender, Classical Quantum Gravity 20, S301 (2003).

[17] R. P. Olling and M. R. Merrifield, Mon. Not. R. Astron. Soc. 326, 164 (2001).

[18] N. J. Cornish, D. N. Spergel, and C. L. Bennett, astro-ph/ 0202001.

[19] N. Seto, S. Kawamura, and T. Nakamura, Phys. Rev. Lett. 87, 221103 (2001).

[20] C. Ungarelli and A. Vecchio, Phys. Rev. D 63, 064030 (2001).

[21] P. L. Bender, Classical Quantum Gravity 21, S1203 (2004).

[22] A. H. Jaffe and D. C. Backer, Astrophys. J. 583, 616 (2003); J. S. B. Wyithe and A. Loeb, Astrophys. J. 590, 691 (2003); A. Sesana, F. Haardt, P. Madau, and M. Volonteri, astro-ph/0401543.

[23] M. Tinto, J.W. Armstrong, and F. B. Estabrook, Phys. Rev. D 63, 021101 (2001).

[24] J. S. Stuart, Science 294, 1691 (2001).

[25] S. Phinney (private communication).

[26] A.W. Adams and J. S. Bloom, astro-ph/0405266. 